\title{
Composición Corporal y Proporcionalidad en Futbolistas Chilenos. Diferencias entre Categorías Juveniles y Campeones Profesionales
}

\author{
Body Composition and Proportionality in Chilean Soccer Players. \\ Differences Between Young and Champion Elite Players
}

Sam Hernández-Jaña ${ }^{1}$; Carlos Jorquera-Aguilera² ${ }^{2}$ Atilio Aldo Almagià-Flores ${ }^{3}$; Rodrigo Yáñez-Sepúlveda ${ }^{4}$ Fernando Rodríguez-Rodríguez ${ }^{1}$

\begin{abstract}
HERNÁNDEZ-JAÑA, S.; JORQUERA-AGUILERA, C.; ALMAGIÀ-FLORES, A. A.; YÁÑEZ-SEPÚLVEDA, R.; RODRÍGUEZRODRÍGUEZ, F. Composición corporal y proporcionalidad en futbolistas chilenos. Diferencias entre categorías juveniles y campeones profesionales. Int. J. Morphol., 39(1):252-259, 2021.
\end{abstract}

RESUMEN: El fútbol es el deporte más popular del mundo, por lo que las variables que benefician el rendimiento y el éxito deportivo generan un gran interés y ocupan un rol esencial en la preparación de futbolistas. Por lo tanto, el objetivo del presente estudio fue analizar la composición corporal y la proporcionalidad según la categoría y posición de juego. Se evaluaron a través de antropometría

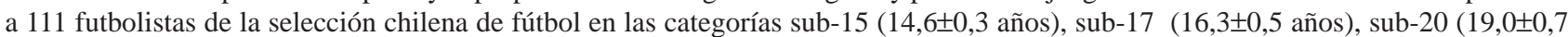

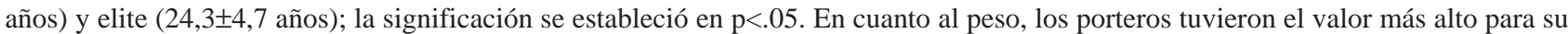
posición; la categoría sub-20 obtuvo el valor más alto $(83,7 \pm 3,8 \mathrm{~kg})$. En cuanto a la estatura, los defensas centrales elite y la categoría sub-17 tuvieron los valores más altos $(187,0 \pm 4,2 \mathrm{~cm}$ y $179,9 \pm 3,5 \mathrm{~cm}$, respectivamente). Los porteros de todas las categorías tienen los mayores valores de IMC, siendo la categoría elite el más alto $\left(25,1 \pm 1,3 \mathrm{~kg} / \mathrm{m}^{2}\right)$. Los delanteros/extremos sub-17 años tienen los valores más altos de masa muscular $(60,5 \pm 8,0 \%)$, mientras que los laterales sub-15 tienen los valores más bajos de masa grasa $(9,6 \pm 0,7 \%)$. Los jugadores de la selección chilena de fútbol tienen valores similares a los de otras investigaciones con jugadores de la misma edad y categoría; sin embargo, las categorías más jóvenes presentan diferencias significativas en las variables que proporcionan ventajas durante un partido con la categoría elite. Por lo tanto, se debe hacer énfasis en equiparar las condiciones físicas antes de promover a un jugador a categorías de mayor edad.

PALABRAS CLAVE: Posición de juego; Fútbol; Antropometría; Masa muscular.

\section{INTRODUCCIÓN}

El fútbol es el deporte más popular en el mundo, involucrando a más de 270 millones de personas en el mundo (Federation Internationale de Football Association, 2007); el éxito indiscutido lo ha posicionado como un deporte que suscita un gran interés científico. El estudio de variables que influyen en el rendimiento deportivo es fundamental, ya que permite mejorar la búsqueda y selección de jugadores jóvenes, otorgando un insumo de gran valor para organizaciones y equipos. Una de las herramientas más usadas debido a su bajo costo, fácil aplicación y relativa precisión es la medición antropométrica, la cual entrega valores de composición corporal y proporcionalidad en diferentes deportes y posiciones en el fútbol (Jorquera et al., 2013; Herdy et al., 2015). Este método permite identificar patrones específicos a través de la edad y de acuerdo con la posición de juego de los deportistas (RodríguezRodríguez et al., 2019); variables como el peso corporal, estatura, masa grasa y masa muscular se han asociado con la velocidad en carrera corta, consumo de oxígeno máximo, salto vertical, entre otros factores determinantes en el éxito deportivo (Wong et al., 2009).

Estudio previos han encontrado diferencias entre posiciones de juego y categorías, especialmente entre ar-

\footnotetext{
${ }^{1}$ Grupo IRyS, Escuela de Educación Física, Pontificia Universidad Católica de Valparaíso, Viña del Mar, Chile.

${ }^{2}$ Escuela de Nutrición y Dietética, Facultad de Ciencias, Universidad Mayor, Santiago, Chile.

${ }^{3}$ Laboratorio de Antropología Física y Anatomía Humana, Institutode Biología, FacultaddeCiencias, Pontificia Universidad Católica de Valparaíso, Valparaíso, Chile.

${ }^{4}$ Escuela de Educación, Pedagogía en Educación Física, Universidad Viña del Mar, Viña del Mar, Chile.
} 
HERNÁNDEZ-JAÑA, S.; JORQUERA-AGUILERA, C.; ALMAGIÀ-FLORES, A. A.; YÁÑEZ-SEPÚLVEDA, R.; RODRÍGUEZ-RODRÍGUEZ, F. Composición corporal y proporcionalidad en futbolistas chilenos. Diferencias entre categorías juveniles y campeones profesionales. Int. J. Morphol., 39(1):252-259, 2021.

queros y delanteros (Gil et al., 2007). Estas variables son decisivas en competiciones y deberían ser tratadas de acuerdo con la posición de juego (Dellal et al., 2010). Por ejemplo, un estudio realizado en futbolistas de la liga inglesa sugirió que el entrenamiento muscular y la reducción de la masa grasa son fundamentales en la preparación y rendimiento deportivo (Sutton et al., 2009). Por lo tanto, cada variable debería ser entrenada según la posición y la función desempeñada por el jugador en el terreno de juego, considerando su edad y nivel deportivo para maximizar cada ventaja que permita beneficiar el juego colectivo, ya que no siempre la mejora individual se traducirá en un beneficio para el equipo (CárdenasFernández et al., 2019).

El conocimiento antropométrico de los campeones continentales es esencial para proyectar a los jugadores jóvenes, especialmente en Chile y en los países latinoamericanos que poseen menos información sobre jugadores con ese nivel y rendimiento deportivo (Rodríguez-Rodríguez et al.). La hipótesis de este estudio fue que la composición corporal y la proporcionalidad son diferentes entre las categorías más jóvenes y la categoría elite; por lo tanto, el presente estudio tuvo como objetivo analizar la composición corporal y la proporcionalidad según la categoría y posición de los jugadores pertenecientes a las categorías sub-15, sub-17, sub-20 y de elite de la selección chilena de fútbol.

\section{MATERIAL Y MÉTODO}

Participantes. Participaron voluntariamente 111 jugadores de la selección chilena (Tabla I); sub-15 ( $\mathrm{n}=27$; 14.6 $\pm 0,3$ años); sub-17 ( $\mathrm{n}=32 ; 16.3 \pm 0,5$ años); sub-20 ( $\mathrm{n}=$ $27 ; 19.0 \pm 0,7$ años $) ; y$ categoría elite $(n=25 ; 24.3 \pm 4.7$ años). Los jugadores elite participaron en la Copa América 2015 y Copa América Centenario 2016 de la CONMEBOL. Todos los jugadores al momento de ser evaluados pertenecían a la selección chilena.

Los jugadores y representantes legales de los menores de edad firmaron voluntariamente un consentimiento informado previo a la participación del estudio. El consentimiento incluía los detalles, objetivos, procedimientos y alcances de la investigación. Además, el estudio se realizó siguiendo las normas éticas internacionales de investigación con seres humanos de la actual Declaración de Helsinki (World Medical Association, 2013). Además, el protocolo fue aprobado por el comité ético-científico de la Universidad Mayor, Chile.
Instrumentos. Los instrumentos antropométricos usados para las mediciones consistieron en un Kit Gaucho Pro "Mercosur"®, fabricado en Argentina bajo la licencia de Rosscraft Canadá para la comercialización en el continente americano (excepto EE. UU., Canadá y México). El kit poseía los siguientes instrumentos: Campbell $20 \AA$ (antropómetro largo), Campbell 10® (antropómetro corto o paquímetro), segmómetro, escuadra, plicómetro o caliper, cinta métrica para perímetros, un estadiómetro para la estatura y una balanza digital Tanita ${ }^{\circledR}$ (Tokio, Japón) para el peso con una precisión de $100 \mathrm{~g}$.

Se utilizó una hoja de datos o proforma digital para registrar la información, siguiendo secuencialmente las posiciones anatómicas de superior a inferior. Las variables que se midieron fueron el peso, estatura, la estatura sentado, el diámetro de seis huesos, diez perímetros (contornos axiales y apendiculares) y ocho pliegues cutáneos.

Procedimientos. Las evaluaciones antropométricas se realizaron durante las primeras horas de la mañana, después del vaciado urinario y con la menor ropa posible para facilitar el marcaje y la evaluación. El protocolo usado fue el recomendado por la International Society for the Advancement of Kinanthropometry (ISAK) (Stewart et al., 2011). Un evaluador nivel III y dos asistentes nivel II de ISAK entrenados previamente en el proceso de evaluación, facilitaron la recolección de datos a través del proceso y la secuencia de mediciones; el error técnico de medición fue de $5 \%$ para pliegues cutáneos y $1 \%$ para perímetros y diámetros de acuerdo con el protocolo ISAK.

La masa muscular fue calculada a través de la fórmula usada por Doupe et al. (1997); la masa grasa según Faulkner (1968) y la masa residual (órganos viscerales y esplácnicos) de acuerdo con Würch (1974). El índice de masa corporal (IMC) fue calculado dividiendo el peso corporal por la estatura en metros al cuadrado (peso [kg]/estatura $[\mathrm{m}]^{2}$ ); la proporcionalidad se calculó a través del modelo teórico phantom según Ross \& Wilson (1974).

Análisis estadístico. El análisis se hizo por posición y categoría. Las variables descriptivas se muestran como media y desviación estándar. Se realizó la prueba ANOVA de un factor para determinar las diferencias entre las categorías y la prueba de Bonferroni para identificar en qué grupos se encontraban. El nivel de significancia se estableció en $\mathrm{p}<0,05$.

Se utilizaron los programas GraphPad Prism versión 8.00 para Mac (GraphPad Software, La Jolla California USA, www.graphpad.com") y SPSS V. 25® (IBM, Chicago, Illinois, USA). 


\section{RESULTADOS}

La Tabla I muestra el promedio de edad y el número de jugadores agrupados por posición y categoría. Los futbolistas fueron agrupados según la edad en 4 categorías (sub-15, sub-17, sub-20 y elite). Además, se analizaron de acuerdo con su posición en el terreno de juego (porteros, laterales, defensas centrales, volantes y delanteros/extremos).

Composición corporal. Las variables analizadas de composición corporal se detallan según categoría y posición (Tabla II):

Porteros. Los porteros no presentan diferencias significativas entre las variables analizadas; sin embargo, el peso ( $p$ 0,093), la masa grasa \% (p 0,056) y la masa residual $\mathrm{kg}(\mathrm{p} 0,093)$ son las más cercanas al valor estadístico esperado $(\mathrm{p}<0,05)$.

Tabla I. Media y desviación estándar de la edad de los futbolistas por posición y categoría.

\begin{tabular}{lccccccc}
\hline Categoría & $\begin{array}{c}\text { Edad } \\
(\text { Media } \pm \text { DE) }\end{array}$ & $\begin{array}{c}\text { Jugador } \\
\text { es }(\mathrm{n})\end{array}$ & PT & LT & DC & VOL & DE \\
\hline Sub-15 & $14.6 \pm 0.3$ & 27 & 4 & 4 & 3 & 12 & 4 \\
Sub-17 & $16.3 \pm 0.5$ & 32 & 2 & 5 & 4 & 14 & 7 \\
Sub-20 & $19.0 \pm 0.7$ & 27 & 4 & 3 & 4 & 12 & 4 \\
Elite & $24.3 \pm 4.7$ & 25 & 3 & 5 & 2 & 9 & 6
\end{tabular}

PT: porteros; LT: laterales; DC: defensas centrales; VOL: volantes; DE: delanteros/ extremos.
Laterales. Los laterales presentaron diferencias significativas en el peso corporal $(\mathrm{p}=0,001)$, estatura $(\mathrm{p}=0,049)$, IMC $(\mathrm{p}=0,002)$, masa muscular $(\mathrm{kg}: \mathrm{p}<0,001 ; \%: \mathrm{p}=$ $0,007)$, masa grasa $\mathrm{kg}(\mathrm{p}=0,017)$ y masa residual $(\mathrm{p}=0,001)$. La mayoría de estas diferencias se hallaron entre las categorías elite y sub-15.

Defensas centrales. Los defensas centrales solo tuvieron una diferencia entre las variables analizadas, siendo la estatura la única significativa $(\mathrm{p}=0,006)$.

Volantes. Los volantes presentaron diferencias significativas en el peso ( $p=0,011)$, estatura $(p=0,016)$, masa muscular $\mathrm{kg}(\mathrm{p}=0,019)$ y masa residual $\mathrm{kg}(\mathrm{p}=0,011)$, encontrándose nuevamente entre las categorías elite y sub-15.

Delanteros/extremos. Los delanteros/extremos tuvieron diferencias en el peso $(\mathrm{p}=0,027), \mathrm{IMC}$ ( $\mathrm{p}$ $=0,032)$, masa muscular $\mathrm{kg}(\mathrm{p}=0,024)$ y masa residual $\mathrm{kg}(\mathrm{p}=0,027)$. Nuevamente las diferencias se hallaron entre las categorías elite y sub- 15 .

La Figura 1 muestra información sobre la composición corporal por categoría y resume gráficamente las diferencias entre ellas.
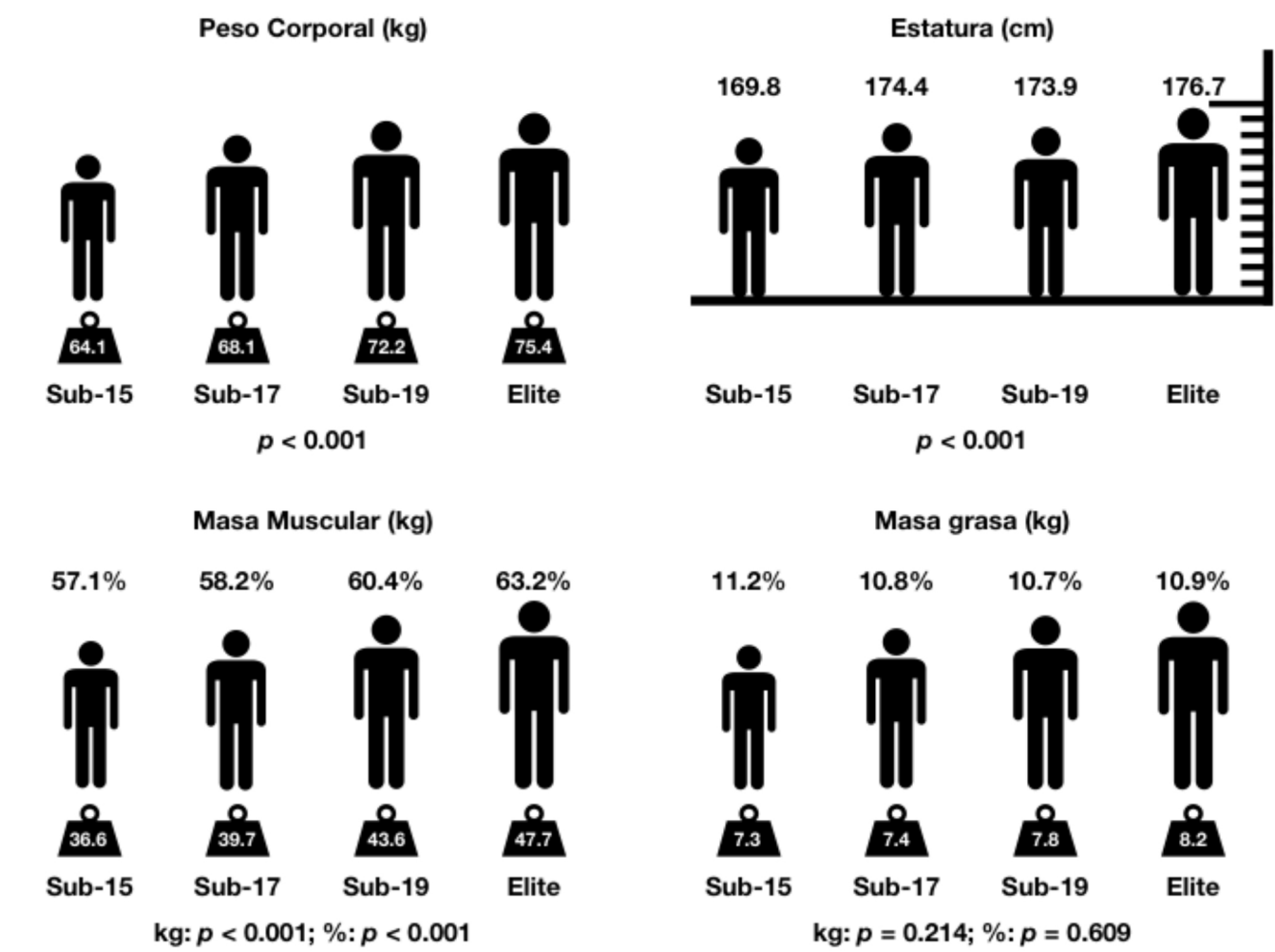

Fig. 1. Representación gráfica de la composición corporal por categoría. 
HERNÁNDEZ-JAÑA, S.; JORQUERA-AGUILERA, C.; ALMAGIÀ-FLORES, A. A.; YÁÑEZ-SEPÚLVEDA, R.; RODRÍGUEZ-RODRÍGUEZ, F. Composición corporal y proporcionalidad en futbolistas chilenos. Diferencias entre categorías juveniles y campeones profesionales. Int. J. Morphol., 39(1):252-259, 2021.

Tabla II. Características de composición corporal y diferencias estadísticas por posición de juego y categoría.

\begin{tabular}{|c|c|c|c|c|c|c|c|c|c|}
\hline \multirow[t]{2}{*}{ Categoría } & \multicolumn{2}{|c|}{ Sub-15 } & \multicolumn{2}{|c|}{ Sub-17 } & \multicolumn{2}{|c|}{ Sub-20 } & \multicolumn{2}{|c|}{ Elite } & \multirow[b]{2}{*}{$p$-valor } \\
\hline & Media & DE & Media & DE & Media & DE & Media & DE & \\
\hline \multicolumn{10}{|c|}{ Porteros $(n=13)$} \\
\hline Peso (kg) & 77.3 & \pm 5.0 & 74.5 & \pm 2.9 & 83.7 & \pm 3.8 & 83.0 & \pm 5.0 & 0.093 \\
\hline Estatura $(\mathrm{cm})$ & 177.5 & \pm 6.2 & 178.6 & \pm 4.4 & 183.3 & \pm 4.3 & 181.8 & \pm 0.8 & 0.349 \\
\hline $\operatorname{IMC}\left(\mathrm{kg} / \mathrm{m}^{2}\right)$ & 24.5 & \pm 0.8 & 23.3 & \pm 0.2 & 24.9 & \pm 1.1 & 25.1 & \pm 1.3 & 0.286 \\
\hline Masa muscular (kg) & 44.7 & \pm 3.2 & 40.5 & \pm 3.1 & 50.0 & \pm 3.0 & 48.3 & \pm 6.8 & 0.109 \\
\hline Masa muscular (\%) & 57.8 & \pm 0.8 & 54.4 & \pm 2.1 & 59.8 & \pm 1.9 & 58.1 & \pm 6.8 & 0.415 \\
\hline Masa grasa (kg) & 10.8 & \pm 1.8 & 8.9 & \pm 0.5 & 11.6 & \pm 2.0 & 8.9 & \pm 0.7 & 0.132 \\
\hline Masa grasa (\%) & 13.9 & \pm 1.6 & 12.0 & \pm 0.2 & 13.9 & \pm 1.8 & 10.7 & \pm 0.9 & 0.056 \\
\hline Masa residual $(\mathrm{kg})$ & 18.6 & \pm 1.2 & 17.94 & \pm 0.7 & 20.2 & \pm 0.9 & 20.0 & \pm 1.2 & 0.093 \\
\hline \multicolumn{10}{|c|}{ Laterales $(n=17)$} \\
\hline Peso (kg) & $\$ \$ \$ 4.2$ & \pm 7.4 & ${ }^{\dagger} 67.4$ & \pm 3.8 & $\div 68.5$ & \pm 6.1 & 75.7 & \pm 5.5 & $* 0.001$ \\
\hline Estatura $(\mathrm{cm})$ & 165.4 & \pm 6.4 & 176.6 & \pm 5.9 & 173.8 & \pm 5.5 & 174.9 & \pm 4.4 & $* 0.049$ \\
\hline $\operatorname{IMC}\left(\mathrm{kg} / \mathrm{m}^{2}\right)$ & $* 19.7$ & \pm 1.5 & ${ }^{*} 21.6$ & \pm 1.3 & 22.7 & \pm 1.9 & † 24.7 & \pm 1.4 & $* 0.002$ \\
\hline Masa muscular $(\mathrm{kg})$ & $\$ \$ 28.4$ & \pm 4.4 & $¥ ¥ 37.2$ & \pm 1.1 & $\doteqdot 39.9$ & \pm 4.9 & 44.3 & \pm 3.4 & $*<0.001$ \\
\hline Masa muscular (\%) & ${ }^{\S} 52.3$ & \pm 1.6 & 55.3 & \pm 2.0 & $\div 58.1$ & \pm 2.1 & $\div 58.6$ & \pm 3.0 & $* 0.007$ \\
\hline Masa grasa $(\mathrm{kg})$ & ${ }^{45} .2$ & \pm 0.8 & 6.9 & \pm 1.1 & 6.9 & \pm 1.1 & $\ddagger 8.3$ & \pm 1.6 & $* 0.017$ \\
\hline Masa grasa (\%) & 9.6 & \pm 0.7 & 10.2 & \pm 1.3 & 10.1 & \pm 0.9 & 10.9 & \pm 1.4 & 0.421 \\
\hline Masa residual $(\mathrm{kg})$ & ${ }^{\ddagger} \$ 11_{3.1}$ & \pm 1.8 & ${ }^{\dagger} 16.2$ & \pm 0.9 & ${ }^{\ddagger} 6.5$ & \pm 1.5 & $\doteqdot 18.2$ & \pm 1.3 & $* 0.001$ \\
\hline \multicolumn{10}{|c|}{ Defensas centrales $(n=13)$} \\
\hline Peso (kg) & 67.0 & \pm 8.2 & 74.3 & \pm 4.6 & 73.3 & \pm 4.2 & 83.3 & \pm 2.8 & 0.055 \\
\hline Estatura $(\mathrm{cm})$ & ${ }^{\sharp 1} 73.4$ & \pm 2.6 & 179.9 & \pm 3.5 & ${ }^{\mathrm{q}} 177.8$ & \pm 2.2 & ॠ1 187.0 & \pm 4.2 & $* 0.006$ \\
\hline $\operatorname{IMC}\left(\mathrm{kg} / \mathrm{m}^{2}\right)$ & 22.3 & \pm 2.9 & 23.0 & \pm 1.5 & 23.2 & \pm 0.9 & 23.9 & \pm 1.9 & 0.827 \\
\hline Masa muscular $(\mathrm{kg})$ & 35.7 & \pm 5.3 & 44.3 & \pm 4.7 & 43.4 & \pm 3.7 & 47.3 & \pm 5.3 & 0.079 \\
\hline Masa muscular (\%) & 53.1 & \pm 1.3 & 59.5 & \pm 4.4 & 59.1 & \pm 2.4 & 56.7 & \pm 4.4 & 0.112 \\
\hline Masa grasa $(\mathrm{kg})$ & 7.9 & \pm 2.6 & 8.4 & \pm 0.7 & 7.9 & \pm 1.0 & 10.1 & \pm 2.2 & 0.459 \\
\hline Masa grasa (\%) & 11.6 & \pm 2.4 & 11.3 & \pm 0.3 & 10.9 & \pm 1.7 & 12.1 & \pm 2.3 & 0.856 \\
\hline Masa residual $(\mathrm{kg})$ & 16.2 & \pm 2.0 & 17.9 & \pm 1.1 & 17.7 & \pm 1.0 & 20.1 & \pm 0.7 & 0.055 \\
\hline \multicolumn{10}{|c|}{ Volantes $(n=47)$} \\
\hline Peso (kg) & ${ }^{*} 6_{3.2}$ & \pm 5.8 & 65.4 & \pm 6.5 & 69.3 & \pm 6.4 & $\doteqdot 71.6$ & \pm 5.1 & $* 0.011$ \\
\hline Estatura $(\mathrm{cm})$ & ${ }^{* 1} 67.4$ & \pm 3.7 & 170.5 & \pm 5.2 & 169.2 & \pm 4.6 & ${ }^{\dagger 1} 73.9$ & \pm 4.0 & $* 0.016$ \\
\hline $\operatorname{IMC}\left(\mathrm{kg} / \mathrm{m}^{2}\right)$ & 22.6 & \pm 1.9 & 22.5 & \pm 2.3 & 24.2 & \pm 1.4 & 23.7 & \pm 1.2 & 0.070 \\
\hline Masa muscular (kg) & 36.1 & \pm 3.9 & 36.3 & \pm 4.8 & 40.3 & \pm 4.3 & 40.4 & \pm 3.5 & $* 0.019$ \\
\hline Masa muscular (\%) & 57.1 & \pm 3.0 & 55.4 & \pm 4.1 & 58.1 & \pm 2.7 & 56.3 & \pm 2.6 & 0.214 \\
\hline Masa grasa (kg) & 7.2 & \pm 1.5 & 7.1 & \pm 1.3 & 7.2 & \pm 1.2 & 7.6 & \pm 1.1 & 0.798 \\
\hline Masa grasa (\%) & 11.3 & \pm 1.2 & 10.8 & \pm 1.2 & 10.3 & \pm 1.0 & 10.6 & \pm 1.1 & 0.213 \\
\hline Masa residual $(\mathrm{kg})$ & ${ }^{*} 1_{5.2}$ & \pm 1.4 & 15.8 & \pm 1.6 & 16.7 & \pm 1.5 & $\ddagger_{7.3}$ & \pm 1.2 & $* 0.011$ \\
\hline \multicolumn{10}{|c|}{ Delanteros/extremos $(n=21)$} \\
\hline Peso (kg) & ${ }^{*} 6.9$ & \pm 1.1 & 69.1 & \pm 6.5 & 71.1 & \pm 9.3 & $7_{4} 4.6$ & \pm 5.7 & $* 0.027$ \\
\hline Estatura $(\mathrm{cm})$ & 171.0 & \pm 4.8 & 176.4 & \pm 3.3 & 175.3 & \pm 6.9 & 176.3 & \pm 3.7 & 0.273 \\
\hline $\operatorname{IMC}\left(\mathrm{kg} / \mathrm{m}^{2}\right)$ & ${ }^{2} 0.9$ & \pm 1.5 & 22.2 & \pm 1.8 & 23.1 & \pm 1.6 & $\doteqdot 24.0$ & \pm 0.9 & $* 0.032$ \\
\hline Masa muscular $(\mathrm{kg})$ & $\div 32.3$ & \pm 1.2 & 41.6 & \pm 5.2 & 43.1 & \pm 7.7 & 42.6 & \pm 4.9 & $* 0.024$ \\
\hline Masa muscular (\%) & 53.0 & \pm 1.1 & 60.5 & \pm 8.0 & 60.3 & \pm 3.5 & 57.1 & \pm 3.2 & 0.152 \\
\hline Masa grasa $(\mathrm{kg})$ & 6.0 & \pm 0.9 & 7.3 & \pm 0.9 & 6.6 & \pm 1.2 & 8.1 & \pm 1.5 & 0.060 \\
\hline Masa grasa (\%) & 9.9 & \pm 1.3 & 10.7 & \pm 1.5 & 9.3 & \pm 0.4 & 10.9 & \pm 1.5 & 0.261 \\
\hline Masa residual $(\mathrm{kg})$ & $\mathrm{x}_{4.7}$ & \pm 0.3 & 16.6 & \pm 1.6 & 17.1 & \pm 2.2 & $\doteqdot 18.0$ & \pm 1.4 & $* 0.027$ \\
\hline
\end{tabular}

*Diferencias significativas; DE: desviación estándar; IMC: índice de masa corporal; Diferencias significativas con Sub-15: †; Sub-17: †; Sub20: ; Elite: ¥.

Análisis de proporcionalidad. El análisis de las mediciones antropométricas proporciona valores cuantificables y comparables entre los grupos por medio del Z-score (Rivera Sosa, 2006) para determinar las diferencias entre los grupos. La Tabla
III muestra los valores de proporcionalidad por categoría, encontrando diferencias significativas en el peso $(p=0,007)$, masa muscular $(\mathrm{p}<0,001)$, diámetro biepicondilar femoral ( $\mathrm{p}$ $=0,024)$ y diámetro del tórax $(\mathrm{p}=0,040)$. 
HERNÁNDEZ-JAÑA, S.; JORQUERA-AGUILERA, C.; ALMAGIÀ-FLORES, A. A.; YÁÑEZ-SEPÚLVEDA, R.; RODRÍGUEZ-RODRÍGUEZ, F. Composición corporal y proporcionalidad en futbolistas chilenos. Diferencias entre categorías juveniles y campeones profesionales. Int. J. Morphol., 39(1):252-259, 2021.

Tabla III. Media, desviación estándar y diferencias estadísticas de proporcionalidad por categoría.

\begin{tabular}{|c|c|c|c|c|c|c|c|c|c|}
\hline \multirow[t]{2}{*}{ Cat egoría } & \multicolumn{2}{|c|}{ Sub-15 } & \multicolumn{2}{|c|}{ Sub-17 } & \multicolumn{2}{|c|}{ Sub-20 } & \multicolumn{2}{|c|}{ Elite } & \multirow[b]{2}{*}{ p-valor } \\
\hline & Media & DE & Media & DE & Media & DE & Media & $\mathrm{DE}$ & \\
\hline Peso (kg) & -0.03 & \pm 0.73 & $\$-0.13$ & \pm 0.72 & 0.35 & \pm 0.52 & 0.32 & \pm 0.44 & $* 0.007$ \\
\hline Masa muscular (kg) & $\$ 3.75$ & \pm 1.40 & $\$ 3.77$ & \pm 1.34 & +5.09 & \pm 1.08 & $\$ 5.68$ & \pm 1.08 & $*<0.001$ \\
\hline Masa grasa $(\mathrm{kg})$ & -1.48 & \pm 0.55 & -1.62 & \pm 0.35 & -1.50 & \pm 0.42 & -1.48 & \pm 0.34 & 0.529 \\
\hline Pliegue abdominal (mm) & -1.55 & \pm 0.73 & -1.87 & \pm 0.45 & -1.78 & \pm 0.72 & -1.89 & \pm 0.47 & 0.141 \\
\hline Perímetro de muslo (cm) & 0.57 & \pm 0.86 & 0.23 & \pm 0.85 & 0.65 & \pm 0.73 & 0.52 & \pm 0.59 & 0.178 \\
\hline Perímetro de pierna $(\mathrm{cm})$ & -0.10 & \pm 0.88 & $\$-0.30$ & \pm 0.75 & ${ }^{\ddagger} 0.19$ & \pm 0.48 & 0.00 & \pm 0.48 & 0.053 \\
\hline Diámetro femoral [biepicondilar] $(\mathrm{cm})$ & $¥ 1.07$ & \pm 0.94 & 0.48 & \pm 0.84 & 0.50 & \pm 0.74 & $\dagger^{0} .24$ & \pm 1.39 & $* 0.024$ \\
\hline Diámetro del tórax (A-P) & 0.86 & \pm 1.01 & $\$ 0.78$ & \pm 0.84 & $\doteqdot 1.43$ & \pm 0.88 & 0.88 & \pm 0.97 & $* 0.040$ \\
\hline
\end{tabular}

DE: desviación estándar; A-P: anteroposterior. *p <0.05. Diferencias significativas con Sub-15: †; Sub-17: †; Sub-20: §; Elite: ¥.

Z-Score Peso Corporal

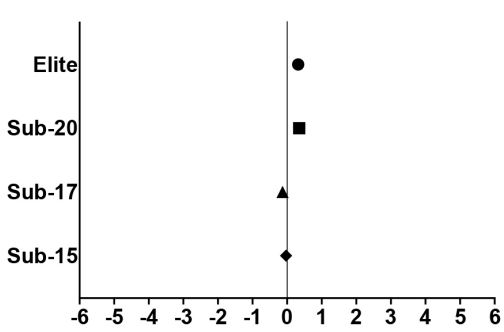

Z-Score Masa Grasa

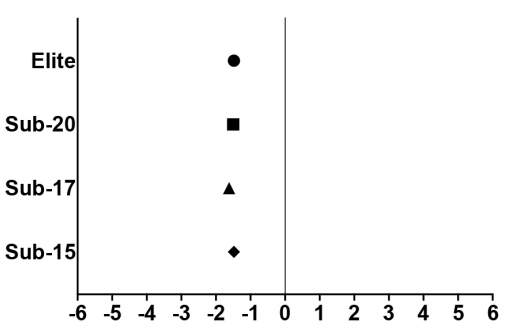

Z-Score Perímetro Muslo

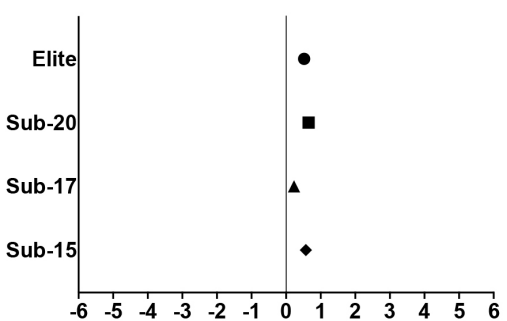

Z-Score Diámetro Femoral

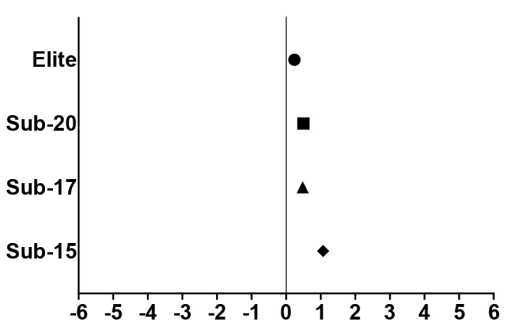

Z-Score Masa Muscular

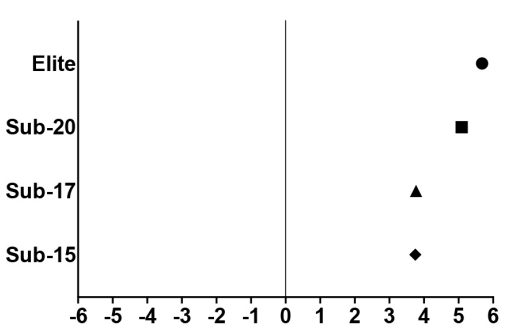

Z-Score Pliegue Abdominal

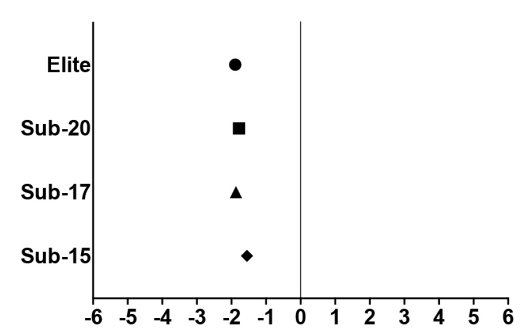

Z-Score Perímetro Pierna

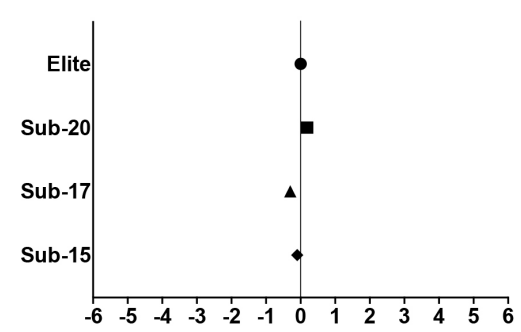

Z-Score Diámetro Tórax (A-P)

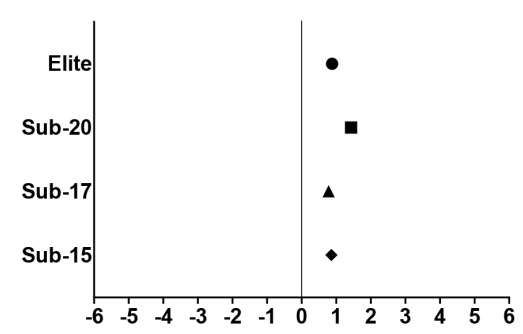

Fig. 2. Proporcionalidad en los valores medios de antropometría por categoría A-P: anteroposterior.

La Figura 2 muestra los valores de los futbolistas evaluados usando la estratagema phantom descrita anteriormente. Las variables más alejadas del modelo de referencia son la masa muscular (elite: $\mathrm{z}$ score =5.68), la masa grasa (sub-17: $\mathrm{z}$ score $=-1,62)$ y el pliegue abdominal (elite: $\mathrm{z}$ score $=-1,89$ ).

\section{DISCUSIÓN}

El objetivo del presente estudio fue analizar la composición corporal y proporcionalidad de acuerdo con la categoría y posición de los futbolistas de la selección chilena. Los principales hallazgos fueron que las categorías sub-15 y sub-17 tienen valores más bajos de peso corporal, estatura y masa muscular y valores más altos de masa grasa que la categoría elite (Campeones Copa América 2015 y Copa América Centenario 2016, CONMEBOL). A pesar de que los resultados podrían predecirse, un gran número de jugadores jóvenes debutan en categorías mayores, por lo que estos valores de referencia sirven para mejorar la toma de decisiones técnicas. Estas diferencias en la composición corporal y proporcionalidad demuestran que las categorías jóvenes están menos aptas para debutar en la categoría elite, a diferencia de la categoría sub-20 que tiene valores similares $y$, en algunas variables, mejores resultados que la categoría elite. 
HERNÁNDEZ-JAÑA, S.; JORQUERA-AGUILERA, C.; ALMAGIÀ-FLORES, A. A.; YÁÑEZ-SEPÚLVEDA, R.; RODRÍGUEZ-RODRÍGUEZ, F. Composición corporal y proporcionalidad en futbolistas chilenos. Diferencias entre categorías juveniles y campeones profesionales. Int. J. Morphol., 39(1):252-259, 2021.

\section{Composición corporal por posición de juego}

Porteros. Los porteros no presentan diferencias estadísticamente significativas en las variables analizadas, lo que sugiere que la edad podría ser un factor menos relevante en el perfil de composición corporal. Sin embargo, se encontró una diferencia de $9.5 \mathrm{~kg}$ de masa muscular entre las categorías sub-17 y sub-20, lo cual representa una ventaja en términos deportivos para esta última categoría. Un alto porcentaje de masa muscular es beneficioso para el desempeño, ya que permite responder a las necesidades de la posición, especialmente cuando una mayor proporción de musculatura se encuentra en las extremidades inferiores (Chelly et al., 2010).

Aunque los valores más altos de peso y estatura son similares a las halladas por Cossio-Bolanos et al. (2012), otros estudios difieren de esos resultados (Herdy et al.; Milsom et al., 2015). Si bien las diferencias no son significativas, existe un delta en el peso corporal de $9.2 \mathrm{~kg}$ entre las categorías mencionadas anteriormente. Estas diferencias en kilogramos son considerables dado el promedio de altura y masa grasa entre ellas, lo que sugiere que las diferencias de peso se deben principalmente a la masa muscular.

Laterales. El grupo más heterogéneo corresponde a los laterales, quienes tuvieron diferencias significativas en el peso, IMC, masa muscular, masa grasa y masa residual. Dado que la función del lateral es defender o, dependiendo del diseño estratégico del juego, desbordar y atacar, se espera que la estatura le permita disputar balones aéreos y apoyar el juego en el ataque. Esta posición es segunda en la distancia total recorrida luego de los volantes (Andrzejewski et al., 2015), lo que hace necesario aumentar las reservas de energía a través del glucógeno muscular y del hígado (Ørtenblad et al., 2013). De hecho, los niveles de glucógeno muscular disminuyen entre $40 \%$ y $90 \%$ durante un partido de fútbol, provocando una disminución en el rendimiento físico de los jugadores(Krustrup et al., 2006).

La categoría sub-17 obtuvo los valores más altos de estatura en esta posición, lo que difiere de los resultados de Lago-Peñas et al. (2011), quienes encontraron valores promedio más bajos en estatura en futbolistas españoles jóvenes. La categoría sub-15 tuvo diferencias significativas con todas las categorías en peso, masa muscular y masa residual, lo que despierta la inquietud sobre las condiciones físicas de jugadores jóvenes que debutan en categorías mayores, arriesgando la integridad física debido a las diferencias en composición corporal y ritmo de juego en el profesionalismo.

Defensas centrales. Los defensas centrales tuvieron una sola diferencia significativa en la estatura, entendiendo la lógica de su función en el terreno de juego. Se espera que estos jugadores sean altos, considerando su permanente juego aéreo defensivo-ofensivo, además, suelen ser los más altos del equipo, tal como lo encontrado en la presente investigación y en estudios previos (Soroka \& Bergier, 2011; Ruas et al., 2015). La lógica de la posición apunta a fortalecer el juego aéreo, dada la tendencia actual en el fútbol de realzar el juego a través de la altura de sus jugadores como una ventaja sobre el oponente.

Volantes. Los volantes presentaron diferencias significativas en peso, estatura, masa muscular y masa residual. Tal como se señaló anteriormente, las diferencias pueden deberse al planteamiento del equipo o al rol del jugador en el terreno de juego, el cual puede variar de acuerdo con los requerimientos del encuentro y del oponente.

Los valores más altos de las cuatro variables con diferencias significativas se encontraron en la categoría elite, la cual tuvo diferencias con la categoría sub-15 en tres variables. Una investigación en futbolistas de la liga iraní encontró valores similares para el peso pero más altos para estatura (Kashani et al., 2013). Otra investigación en jugadores croatas y de Emiratos Árabes Unidos halló valores similares para la estatura pero más bajos para el peso (Sporis et al., 2009; Magalhães Sales et al., 2014); esta variabilidad podría deberse al rol del jugador, quien puede desempeñarse por el centro o por las bandas del terreno de juego. De hecho, esta posición puede tomar diferentes funciones durante el juego, ya que son los volantes centrales quienes han registrado la mayor distancia recorrida en comparación al resto del equipo (Soroka \& Bergier; Andrzejewski et al.).

Delanteros/Extremos. Los delanteros/extremos presentaron diferencias significativas en peso, IMC, masa muscular y masa residual, las cuales se encontraron específicamente entre las categorías elite y sub-15. Los valores de peso de la categoría elite son similares a los hallados por Kashani et al., en futbolistas profesionales de la liga iraní. Respecto del IMC, los valores fueron similares a los encontrados por Joksimovic et al. (2009), en jugadores austriacos y suizos. Los valores más altos de masa muscular se hallaron en la categoría sub-20, los cuales fueron más altos que los registrados por Herdy et al.; sin embargo, la masa residual se mantuvo igual en el mismo estudio y categoría. Lo anterior, al igual que con los laterales, deja manifestada la inquietud sobre la preparación y características físicas apropiadas de jugadores de categorías menores que debutan o entrenan con las categorías mayores.

Proporcionalidad. Los valores de proporcionalidad sobre las características físicas de los jugadores se muestran por 
categoría. La masa muscular, masa grasa y pliegue abdominal fueron los valores más alejados del modelo de referencia.

La masa muscular presentó valores positivos, mientras que la masa grasa presentó valores negativos en todas las categorías, tal como los encontrados por Hencken \& White (2006), quienes hallaron valores con el mismo comportamiento respecto del phantom, reforzando la comprensión de cómo el deporte influye en la composición corporal de los futbolistas. Por otra parte, el pliegue abdominal también se alejó del modelo, estableciendo valores negativos para todas las categorías, tal como los obtenidos por Jorquera et al. (2013), en futbolistas chilenos; este último estudio, también encontró valores positivos en el diámetro anteroposterior del tórax y el perímetro de la pierna, tal como la presente investigación. El diámetro del tórax está relacionado con la función pulmonar (Erceg et al., 2011), la cual impacta directamente el desempeño deportivo (Nicks et al., 2009), mientras que un bajo valor en el perímetro de la pierna ayuda en la economía de la carrera (Eksterowicz et al., 2016). Lo anterior toma sentido cuando se analizan las distancias recorridas durante un partido de fútbol, ya que los esfuerzos de baja intensidad predominan por sobre los de alta intensidad (Andrzejewski et al.).

Variables como la composición corporal y la antropometría son indicadores apropiados sobre el desempeño deportivo en divisiones inferiores. Esto es útil cuando se proyectan jugadores hacia el alto rendimiento o a programas de detección de talentos, por lo que debería considerarse que muchas de estas variables antropométricas pueden ser entrenadas. De esta manera, podrían complementarse otras evaluaciones para la detección de talentos (Dodd \& Newans, 2018). La estatura, porcentaje de masa muscular y masa grasa se han convertido en variables determinantes en el fútbol y otros deportes de oposición, debido a que proveen ventajas considerables. Mientras que los jugadores altos suelen ganar las disputas de los balones aéreos, un futbolista bien entrenado podría igualar la disputa a través de la explosividad y altura de salto (Kraska et al., 2009). La masa muscular beneficia el juego uno contra uno al dar ventaja al jugador más fuerte (Jorquera et al., 2012), mientras que valores bajos de masa grasa tienen un impacto en la aceleración y el desempeño en el salto (Dodd \& Newans).

En conclusión, este estudio encontró importantes diferencias en el análisis de composición corporal y la proporcionalidad entre las categorías más jóvenes (sub-15 and sub-17) y la categoría sub-20 y elite (Campeones CONMEBOL Copa América 2015-2016).

Si bien las habilidades técnicas son importantes, estas características físicas ayudan a desarrollar aspectos es- pecíficos del deporte. Por otra parte, el peso, la estatura y la masa muscular más baja pone en desventaja a jugadores jóvenes y aumenta el riesgo de lesiones. Por lo tanto, los equipos deberían considerar que esas características físicas deben desarrollarse antes de tomar la decisión de que un jugador haga su debut en categorías mayores, de lo contrario su carrera deportiva podría ser breve.

\section{AGRADECIMIENTOS}

Queremos agradecer a los jugadores de fútbol, equipos, entrenadores y directivos del campeonato nacional. También, a los estudiantes de Nutrición y Dietética de la Universidad Mayor que midieron las variables antropométricas.

HERNÁNDEZ-JAÑA, S.; JORQUERA-AGUILERA, C.; ALMAGIÀ-FLORES, A. A.; YÁÑEZ-SEPÚLVEDA, R.; RODRÍGUEZ-RODRÍGUEZ, F. Body composition and proportionality in Chilean soccer players; Differences between young and champion elite players. Int. J. Morphol., 39(1):252259,2021

SUMMARY: Soccer is the most popular sport in the world. Thus, the variables that benefit performance and success have sparked great interest and occupy an essential role in the preparation of athletes. Therefore, the aim of the present study was to analyze the body composition and proportionality according to categories and playing position. 111 male soccer players of the Chilean national soccer team under-15 (14.6 \pm 0.3 years), under-17 (16.3 \pm 0.5 years), under-20 ( $19.0 \pm 0.7$ years), and elite $(24.3 \pm 4.7$ years $)$ categories were evaluated through anthropometry. Values of body composition and proportionality were recorded and organized by position and category; significance was established at $\mathrm{p}<.05$. For weight, goalkeepers had the highest for their position; under-20 players, the highest for their category $(83.7 \pm 3.8 \mathrm{~kg})$. Regarding height, elite and under-17 central defenders had the highest values (187.0 \pm 4.2 $\mathrm{cm}$ and $179.9 \pm 3.5 \mathrm{~cm}$, respectively). Goalkeepers in all categories have the highest BMI values; the highest were observed in elite $(25.1 \pm 1.3 \mathrm{~kg} / \mathrm{m} 2)$. Under-17 forwards/extremes had the highest values in terms of muscle mass percentage $(60.5 \pm 8.0 \%)$, whereas under-15 fullbacks have the lowest values in terms of fat mass $(9.6 \pm 0.7 \%)$. The players of the Chilean national soccer team have similar values to other researches with players of the same age and category; however, the younger categories have significant differences in the variables that provide advantages during a game with the elite category. Therefore, emphasis should be placed on equating physical conditions before promoting a player to the older categories.

KEY WORDS: Game position; Football, anthropometry; Muscle mass. 


\section{REFERENCIAS BIBLIOGRÁFICAS}

Andrzejewski, M.; Chmura, J.; Pluta, B. \& Konarski, J. M. Sprinting activities and distance covered by top level Europa league soccer players. Int. J. Sports Sci. Coach., 10(1):39-50, 2015.

Cárdenas-Fernández, V.; Chinchilla-Minguet, J. L. \& Castillo-Rodríguez, A. Somatotype and body composition in young soccer players according to the playing position and sport success. J. Strength Cond. Res., 33(7):1904$11,2019$.

Chelly, M. S.; Chérif, N.; Amar, M. B.; Hermassi, S.; Fathloun, M.; Bouhlel, E.; Tabka, Z. \& Shephard, R. J. Relationships of peak leg power, 1 maximal repetition half back squat, and leg muscle volume to 5-m sprint performance of junior soccer players. J. Strength Cond. Res., 24(1):26671, 2010,

Cossio-Bolanos, M.; Portella, D.; Hespanhol, J. E.; Fraser, N. \& De Arruda, M. Body size and composition of the elite Peruvian soccer player. $J$. Exerc. Physiol., 15(3):30-8, 2012.

Dellal, A.; Wong, D. P. \& Moalla, W. Physical and technical activity of soccer players in the French First League - with special reference to their playing position. Int. Sport Med. J., 11(2):278-90, 2010,

Dodd, K. D. \& Newans, T. J. Talent identification for soccer: Physiological aspects. J. Sci. Med. Sport, 21(10):1073-8, 2018.

Doupe, M. B.; Martin, A. D.; Searle, M. S.; Kriellaars, D. J. \& Giesbrecht, G. G. A new formula for population-based estimation of whole body muscle mass in males. Can. J. Appl. Physiol., 22(6):598-608, 1997.

Eksterowicz, J.; Napiera?a, M. \& Z ukow, W. How the Kenyan runner's body structure affects sports results. Hum. Mov., 17(1):8-14, 2016.

Erceg, M.; Jelaska, I. \& Males`, B. Ventilation characteristics of young soccer players. Homo Sport., 13(2):7-11, 2011.

Faulkner, J. A. Physiology of Swimming and Diving. En: Falls, H. (Ed.). Exercise

Federation Internationale de Football Association (FIFA). FIFA Big Count 2006: 270 million people active in football. FIFA Communications Division, Information Services, 2007. Disponible en: https:// resources.fifa.com/image/upload/big-count-estadisticas520058.pdf?cloudid=mzid0qmguixkcmruvema\#: :text=There $\% 20$ are $\% 20$ now \%20265\%20million, of \%20its \%20207\%20member $\% 20$ associations.

Gil, S. M.; Gil, J.; Ruiz, F.; Irazusta, A. \& Irazusta, J. Physiological and anthropometric characteristics of young soccer players according to their playing position: relevance for the selection process. J. Strength Cond. Res., 21(2):438-45, 2007.

Hencken, C. \& White, C. Anthropometric assessment of Premiership soccer players in relation to playing position. Eur. J. Sport Sci., 6(4):205-11, 2006.

Herdy, C. V.; Nunes, R. A. M.; Junior, R. F. M.; Rodríguez, F. R.; Mattos, D. S.; Ramos, S.; Teixeira, R.; Costa e Silva, G. \& Novaes, J. S. Perfil antropométrico, composición corporal y somatotipo de jóvenes futbolistas brasileños de diferentes categorías y posiciones. Educ. Fis. Dep., 34(2):507-24, 2015.

Joksimovic', A.; Smajic', M.; Molnar, S. \& Stankovic', D. An analysis of anthropomorphological characteristics of participants in the 2008 European Football Championship. Serbian J. Sports Sci., 3(2):71-9, 2009.

Jorquera, A. C.; Rodríguez, R. F.; Torrealba, V. M. I. \& Barraza, G. F. Body Composition and Somatotype of Chilean Soccer Players Sub 16 y Sub 17. Int. J. Morphol., 30(1):247-52, 2012.

Jorquera, A. C.; Rodríguez, R. F.; Torrealba, V. M. I.; Campos, S. J.; Gracia, L. N. \& Holway, F. Anthropometric characteristics of Chilean professional football players. Int. J. Morphol., 31(2):609-14, 2013.

Kashani, A.; Khotbesara, R. D.; Maskooni, M. D. \& Djafarian, K. Body composition of elite Iranian soccer players according to the playing position. Int. Res. J. Appl. Basic Sci., 7(14):1184-8, 2013.

Kraska, J. M.; Ramsey, M. W.; Haff, G. G.; Fethke, N.; Sands, W. A.; Stone, M. E.\& Stone, M. H. Relationship between strength characteristics and unweighted and weighted vertical jump height. Int. J. Sports Physiol. Perform., 4(4):461-73, 2009.
Krustrup, P.; Mohr, M.; Steensberg, A.; Bencke, J.; Kjaer, M. \& Bangsbo, J. Muscle and blood metabolites during a soccer game: implications for sprint performance. Med. Sci. Sports Exerc., 38(6):1165-74, 2006.

Lago-Peñas, C.; Casais, L.; Dellal, A.; Rey, E. \& Domínguez, E. Anthropometric and physiological characteristics of young soccer players according to their playing positions: relevance for competition success. J. Strength Cond. Res., 25(12):3358-67, 2011.

Magalhães Sales, M.; Vieira Browne, R. A.; Yukio Asamo, R.; Olher, R. R. V.; Vila Nova, J. F.; Moraes \& Simões, H. G. Physical fitness and anthropometric characteristics in professional soccer players of the United Arab Emirates. Rev. Andal. Med. Deporte, 7(3):106-10, 2014.

Milsom, J.; Naughton, R.; O’Boyle, A.; Iqbal, Z.; Morgans, R.; Drust, B. \& Morton, J. P. Body composition assessment of English Premier League soccer players: a comparative DXA analysis of first team, U21 and U18 squads. J. Sports Sci., 33(17):1799-806, 2015.

Nicks, C. R.; Morgan, D. W.; Fuller, D. K. \& Caputo, J. L. The influence of respiratory muscle training upon intermittent exercise performance. Int. J. Sports Med., 30(1):16-21, 2009.

Ørtenblad, N.; Westerblad, H. \& Nielsen, J. Muscle glycogen stores and fatigue. J. Physiol., 591(Pt. 18):4405-13, 2013.

Physiology. Baltimore, Academic Press, 1968. pp.87-95.

Rivera Sosa, J. M. Valoración del somatotipo y proporcionalidad de futbolistas universitarios Mexicanos respecto a futbolistas profesionales. Rev. Int. Med. Cienc. Act. Fis. Deporte, 6(21):16-28, 2006.

Rodríguez-Rodríguez, F.; López-Fuenzalida, A.; Holway, F. \& Jorquera Aguilera, C. Diferencias antropométricas por posición de juego en futbolistas profesionales chilenos. Nutr. Hosp., 36(4):846-53, 2019.

Ross, W. D. \& Wilson, N. C. A stratagem for proportional growth assessment. Acta Paediatr. Belg., 28 Suppl.:169-82, 1974.

Ruas, C. V.; Minozzo, F.; Pinto, M. D.; Brown, L. E. \& Pinto, R. S. Lowerextremity strength ratios of professional soccer players according to field position. J. Strength Cond. Res., 29(5):1120-6, 2015.

Soroka, A. \& Bergier, J. The relationship among the somatic characteristics, age and covered distance of football players. Hum. Mov., 12(4):353-60, 2011.

Sporis, G.; Jukic, I.; Ostojic, S. M. \& Milanovic, D. Fitness profiling in soccer: physical and physiologic characteristics of elite players. J. Strength Cond. Res., 23(7):1947-53, 2009.

Stewart, A.; Marfell-Jones, M.; Olds, T. \& De Ridder, J. International Standards for Anthropometric Assessment. Potchefstroom, ISAK, 2011.

Sutton, L.; Scott, M.; Wallace, J. \& Reilly, T. Body composition of English Premier League soccer players: influence of playing position, international status, and ethnicity. J. Sports Sci., 27(10):1019-26, 2009.

Wong, P. L.; Chamari, K.; Dellal, A. \& Wisløff, U. Relationship between anthropometric and physiological characteristics in youth soccer players. J. Strength Cond. Res., 23(4):1204-10, 2009.

World Medical Association. World Medical Association Declaration of Helsinki: ethical principles for medical research involving human subjects. JAMA, 310(20):2191-4, 2013.

Würch, A. La femme et le sport. Med. Sport Fr., 4:441-5, 1974.

\section{Dirección para correspondencia \\ Sam Hernández Jaña \\ Avenida El Bosque 1290 \\ Viña del Mar \\ Valparaíso - CHILE}

Email: sam.hernandez.jana@gmail.com

Recibido: $24-08-2020$

Aceptado: 17-09-2020 